\title{
science \\ The first year of the Crossref Ambassador Program: highlights and challenges
}

\author{
Vanessa Fairhurst \\ Crossref, Oxford Centre for Innovation (OCFI), Oxford, UK
}

\section{Introduction}

'Crossref makes research outputs easy to find, cite, link, and assess. We're a not-for-profit membership organization that exists to make scholarly communications better' [1].

Crossref was founded in 2000 by a group of 12 publishers who wanted to find a way in which to effectively solve the issue of link-rot for citations and simplify the process of linking to research on other publisher platforms. Prior to Crossref, publishers needed to make individual agreements with one another in order to link to articles on other publisher's websites, something which was essential given the fact that a references section in any one academic article can contain links to a number of articles from different publishers. Whilst manageable among a small group of publishers, this does not scale, and so Crossref, a not-for-profit, neutral, membership organization, was formed to make research outputs easier to find, cite, link and assess, providing open infrastructure for the scholarly community.

The ability to assign DOIs to content is still arguably the main reason organizations join Crossref. A DOI is a persistent link, expressed as a URL, which when clicked upon redirects to the publisher landing page where the reader can then gain access to that content. However, there is much more to Crossref than DOIs, they also hold an immense store of metadata for over 100 million scholarly content items [2]. When a member registers a piece of content with us, the DOI is only one part of the vast array of metadata that can be associated with it. Other vital information such as article title, author names, and identifiers (ORCID iDs), publication dates, affiliations, license information, abstracts, references, text mining URLs and data sets, and more, can be included. The more comprehensive the metadata that is deposited with Crossref, the more discoverable the piece of content is. Metadata is not only important for driving traffic to publications, but it can be used for a wide range of different purposes such as

Received: February 4, 2019 Accepted: February 7, 2019

Correspondence to Vanessa Fairhurst vfairhurst@crossref.org

ORCID

Vanessa Fairhurst

https://orcid.org/0000-0001-8511-8689 citation matching, tracking funded research outputs, in annotation tools, metrics, indexing services and library discovery systems to name but a few (Fig. 1).

Crossref also provides a variety of additional services which have been expanded and developed over the years with an increase in members and the collaborative power of their metadata. Services such as Crossmark enables publishers to display if content has been updated, corrected or retracted, whereas the Similarity Check service helps editors to ensure editorial originality, and the Event Data service captures where research is discussed and shared online. 


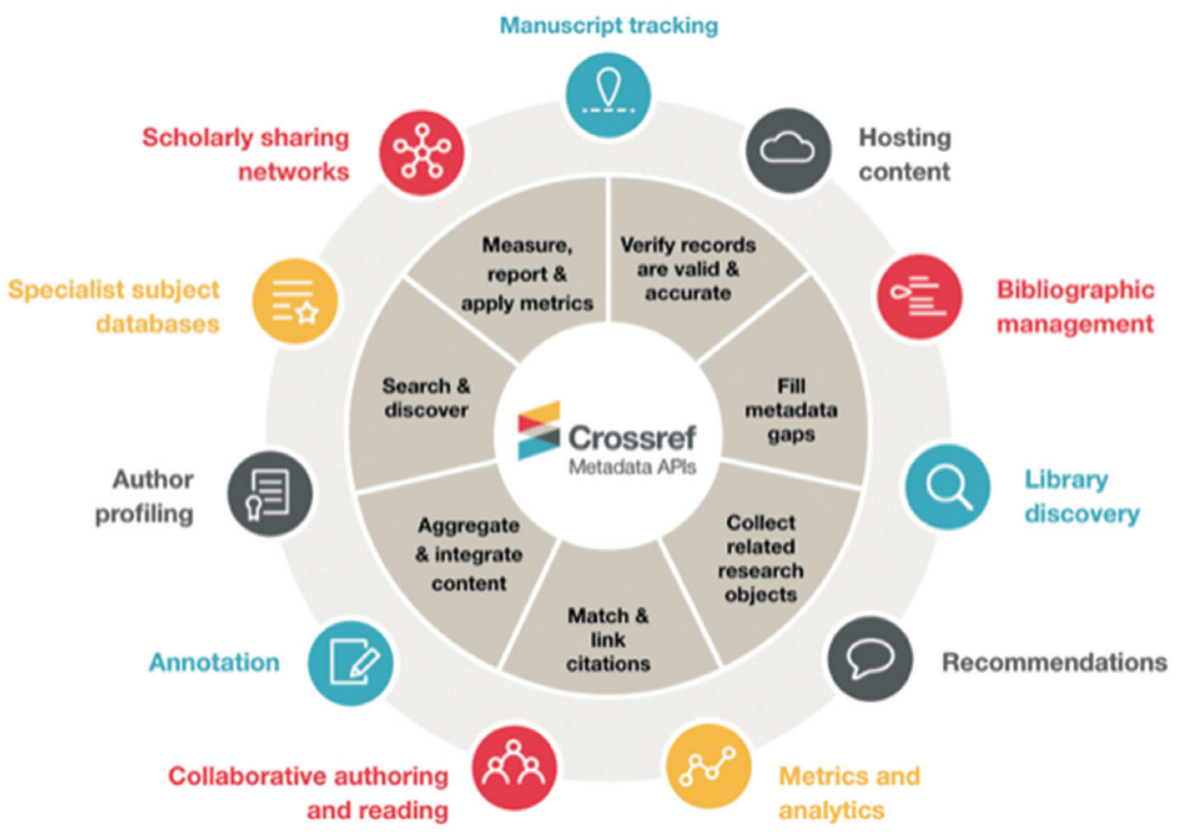

Fig. 1. The uses of Crossref metadata. API, application programming interface.

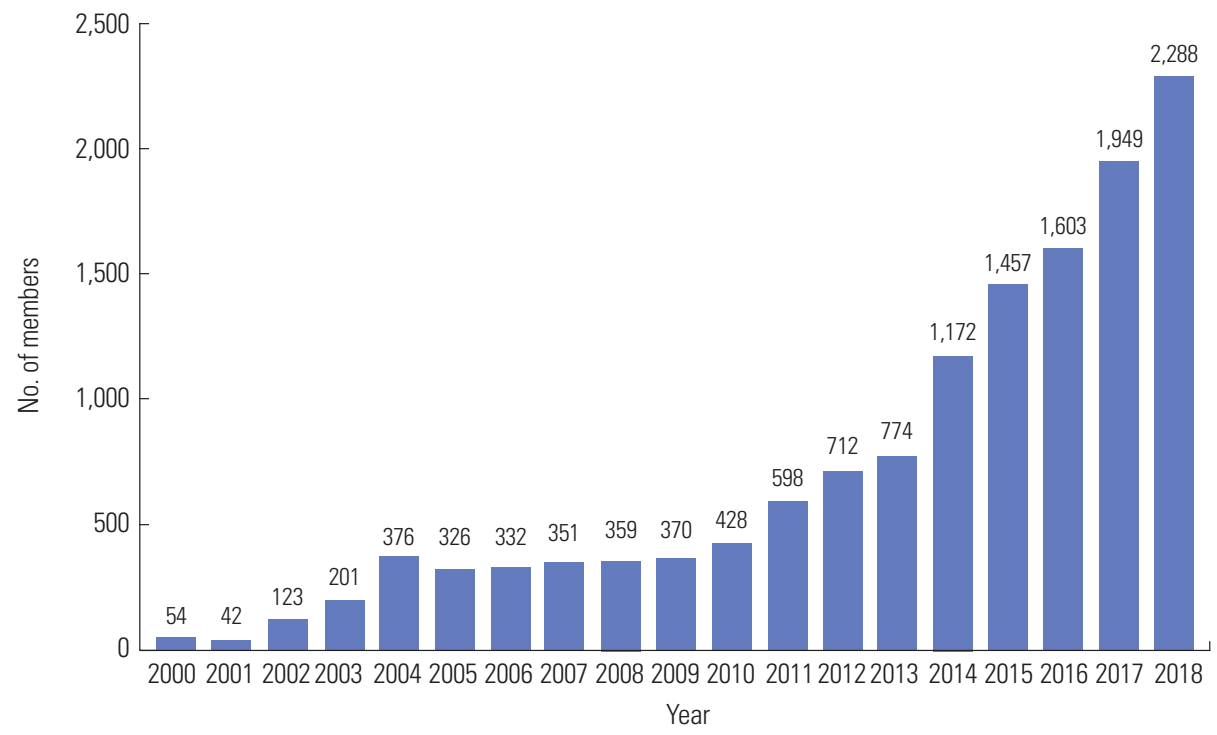

Fig. 2. Crossref members joining per year.

From humble beginnings of 54 members at the end of the first year, Crossref membership has grown substantially to over 11,000 members from 128 countries around the world and continues to grow year on year. Crossref therefore plays a vital role in ensuring linkages between global research outputs continue to be made and built upon (Fig. 2).

\section{The Rationale behind the Ambassador Program}

As of January 2019, Crossref has 38 members of staff [3] based primarily in Boston, US and Oxford, UK. The team is constantly striving for ways in which to meet the varied needs of their global and ever-increasing community and provide the levels of support that members require.

Feedback from members identified a need for greater sup- 


\section{science editing/}

port in different languages and time-zones. Members would like to see representatives from Crossref in their region, who could be present at industry events and to answer queries and provide information in non-English languages. This is particularly pertinent given that feedback from the community has shown that some members struggle with the technical aspects of membership, particularly when working with English as a second language. Additionally, as an innovative organization, new iterations and improvements to existing tools and services are constantly being developed, as such information and advice on best practice can get out-of-date quite quickly and it is important to provide members with both up-to-date and accurate information.

Having a wider spread of Crossref representatives around the world is not only incredibly useful in terms of dissemination of accurate and accessible information, but also to provide greater insight to Crossref of the different cultural, legal, technological, structural and social challenges members face in their countries.

Crossref also recognized that a number of organizations and individuals around the world were already working within their communities to solve these issues and provide support to others in joining and participating in Crossref. This included conducting training sessions, translating Crossref materials, and holding webinars in local languages. The Ambassador Program was therefore conceived as a way in which to empower and formally recognize those who already work with Crossref and help them to provide the best level of support in their communities.

In addition to feedback from their own community, further research was undertaken into what similar programs already exist within the wider scholarly research industry and what levels of success they have had. This also included discussions with other organizations about their experiences and challenges with running their own ambassador programs.

Following these internal and external conversations at Crossref, the team identified several priorities that the Ambassador Program should address: (1) to gain a deeper understanding of certain audiences or countries, (2) to increase outbound education, with both existing members and new audiences, (3) to improve communication with and between nonEnglish speaking communities, (4) to empower Crossref members to help and advise one another, and (5) to help people to further their relationships with Crossref, deepen their knowledge, and aid their professional development.

\section{The First Year of the Ambassador Program}

Over the course of the first year, Crossref has recruited 16 new Ambassadors based in Australia, Brazil, Colombia, India,

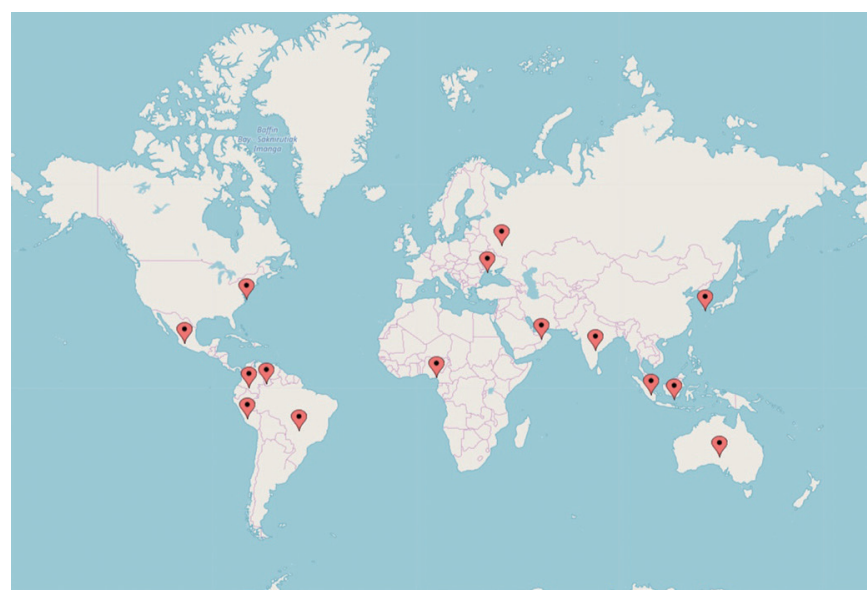

Fig. 3. Geographical spread of Crossref Ambassadors, January 2019. Map created using MapCustomizer.com (https://www.mapcustomizer.com).

Indonesia, Mexico, Nigeria, Peru, Russia, Singapore, South Korea, UAE, Ukraine, USA, and Venezuela (Fig. 3) [4].

It was decided to keep the Ambassador Program small with 10 to 20 individuals in the first year at least. The total number envisioned has not been agreed upon but is likely not to exceed 50. The reason for this is to ensure that Crossref can build personal relationships with the Ambassador team, be on hand to answer questions that arise, help provide training and other support, and to enable the Ambassadors to build relationships with the other volunteers in the team.

Ambassadors are identified via existing working relationships with Crossref, or via the online application form (https:// www.crossref.org/community/ambassadors/). The form allows an individual to make an expression of interest and asks some basic questions regarding their understanding of Crossref, their work preferences, areas they would like more knowledge of, as well as some more general information about themselves. Completion of the application form is not automatic approval to become an ambassador. The forms are reviewed internally, and a call is then scheduled to discuss the role and the individual's fit in more depth. With aiming at keeping the program small, it is important to select the correct people with a good level of knowledge of Crossref and the scholarly research industry, who are well connected, and passionate about the work that Crossref does.

After the initial selection process and introductory call, a group training session is scheduled. Although all Ambassadors have some level of familiarity with Crossref services and processes, this can vary greatly depending on their role and exposure to Crossref in their professional lives. The introductory training session not only provides a basic overview of Crossref, but it also helps to highlight any gaps in knowledge and areas for further training or ways in which the on-boarding process 


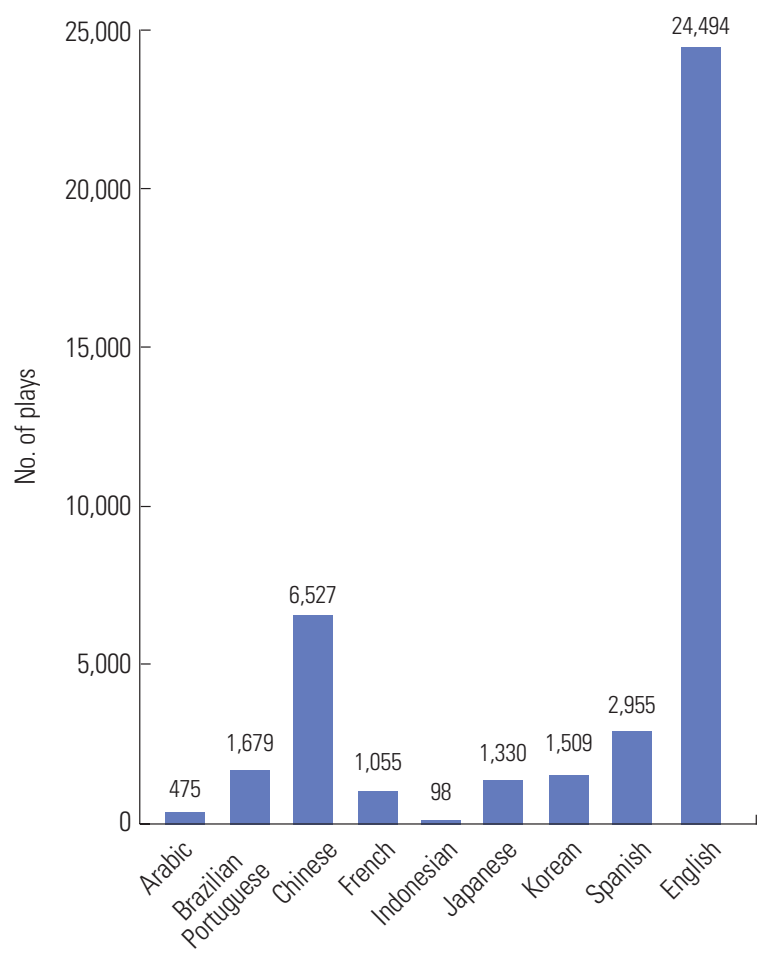

Fig. 4. Crossref service video plays per language.

can be improved. Ambassadors are also encouraged to contact the Crossref team as necessary and on-going check-ins and reviews ensure that the volunteers are happy with their level of support and provide a space to voice any concerns.

\section{Key Highlights}

Over the course of 2018, there were a number of key achievements which would not have been possible without the assistance of the Ambassador team. A big focus was to provide support in a wider array of languages. Ambassadors helped translate presentations, messaging, promotional and educational materials. There are now videos available for each of the Crossref services in nine languages including English, French, Spanish, Brazilian Portuguese, Chinese, Japanese, Korean, Arabic, and Bahasa Indonesia [5]. Many Ambassadors were actively involved in the production of these videos either by providing translation or proofreading services or by helping to promote the videos via social media and within their own networks once completed (Fig. 4).

Crossref has also been able to run a successful series of webinars in Russian, Brazilian Portuguese, Spanish and Arabic [6]. Several Ambassadors have taken a lead in running these webinars in their own languages with assistance from Crossref staff on producing materials and answering questions on

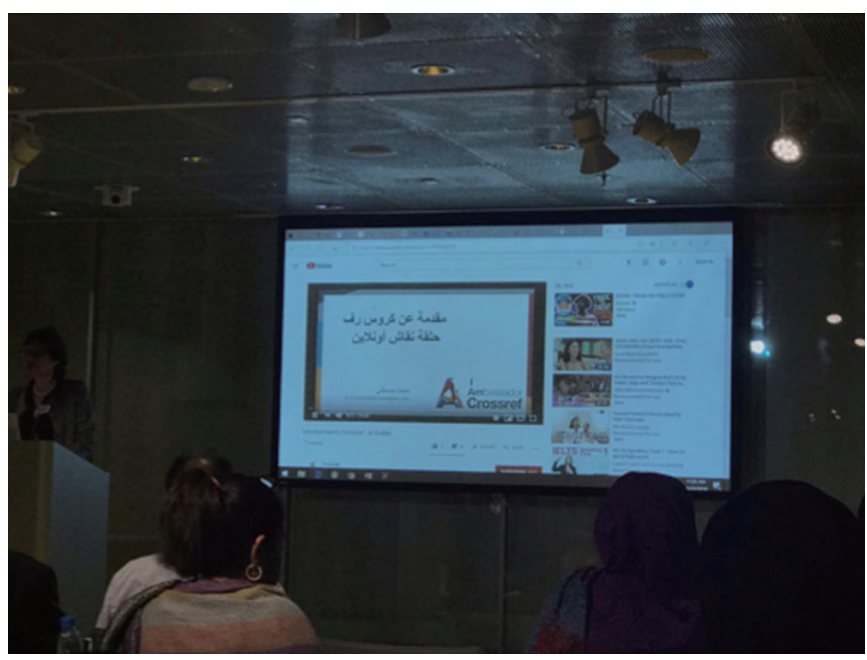

Fig. 5. Arabic webinars presented at Qatar National Library during an Open Access Week workshop.

the day. The webinars have been well attended on the day, as well as many members choosing to watch the recordings at their own leisure (Fig. 5).

Another of the ways in which Crossref has been expanding its international outreach activities is to hold a series of oneday 'LIVE local' events, in different locations around the world, providing information on developments and an opportunity to meet members face-to-face. In 2018 Crossref held events in Japan, South Africa, Russia, Germany, Brazil and India [7]. Ambassadors provide valuable help in organising regional events such as these from recommendations on venues, accommodation, travel and potential guest speakers, to attending and presenting at the events themselves. In addition to these events, Ambassadors are able to represent Crossref at other related industry events in their regions. Korean Ambassador Jae Hwa Chang attended ISMTE in Singapore, Edilson Damasio presented at SNBU and SciELO conferences in Brazil, and Arley Soto assisted at a Redalyc workshop in Peru. This enables greater reach and exposure of Crossref services and provides Ambassadors with a platform and the formal recognition to speak on behalf of the organization.

As Ambassadors are often interacting with the wider community as official representatives of Crossref, it is also important that they are kept up to date with new developments, and that they get to take a first look at new products or services. The ambassador team has participated in beta-testing of a number of new initiatives including the new Metadata Manager and Participation Reports tools and the upcoming Community Forum. By providing feedback from their own user perspective and from how they anticipate those in their communities will view and use these tools, it enables Crossref to make any changes before wider release. 


\section{Challenges and Opportunities}

There have of course been challenges as well as successes over the course of the first year, as with any new initiative in an experimental phase. The Ambassador Program was designed with flexibility in mind, due to the voluntary nature of the role. Crossref also wanted to ensure that ambassadors enjoy the work that they do, that it compliments their existing professional roles and that they contribute to the program in ways that fit their skill set, interests and availability. Of course, this does mean that some ambassadors have had more opportunity to be involved with the work of Crossref than others within this first year. This is not necessarily a problem; however, it is important to manage expectations and ensure that everyone has the opportunity to participate fully, as well as maintaining that work is evenly spread and none feel overburdened. This is particularly important in regions where there is only one ambassador, or few with proficiency in a particular language.

Ambassadors have expressed a wish for further training materials, both for themselves to deepen their knowledge of Crossref, and to provide to members who approach them with questions and requests for information. This will also include translating more support documentation into other languages to make this accessible for a wider audience. In addition to underscoring the need for more multi-lingual support, the program has also highlighted the complexity of much of the current English support documentation. The breadth of activities Crossref enables members to achieve is valuable, however, this abundance of uses can cause some, especially those who have just joined, to become confused by how to participate and what obligations they need to fulfill. Therefore, there is a need to provide well-structured and simple English language support documentation.

Another somewhat unavoidable challenge for the Ambassador Program is on how to ensure the ambassadors build working relationships with one-another as well as with the Crossref team. Something which would aid them not only personally, but professionally, in making wider industry connections. Such a large geographical spread is essential for being able to reach a large, international audience, but makes meeting in person quite difficult with issues around cost, visas, and travel time. Online meeting software greatly helps with providing a cost-efficient and alternative way to meet, however still presents challenges in terms of time-zones. Additionally, although keeping the Ambassador Program small has benefits in terms of building interpersonal relationships, some countries, such as Brazil to take one such example, can be vast. There are issues of inter-country travel to take into consideration and Crossref may need to assess if more representatives are required in certain regions.

\section{Future Plans}

Initial feedback on the program has been overwhelmingly positive, both from the ambassador team themselves and the wider Crossref community. Crossref plan on expanding the number of ambassadors in 2019 and have already received a number of expressions of interest from individuals also interested in learning more and joining the team. Again, there is a need to be selective and ensure that new ambassadors possess the right skills, knowledge and time to dedicate to the role.

In early 2019 Crossref will be launching an online Community Forum. This will provide an open platform where members can pose questions to Crossref staff or to one another, participate in discussions, provide feedback, and share ideas and recommendations. This space will also provide members with the opportunity to post queries in their native language and receive responses from the group in that same language. Ambassadors will be actively engaged in the platform, both in answering queries and in the moderation of non-English content. The community forum will also have a dedicated space for ambassadors to discuss, share their plans and activities, and to facilitate relationship building within the team.

In response to feedback from ambassadors, further training will be provided on technical processes at Crossref and on how to clearly communicate Crossref's role and significance in the scholarly research space. A priority will be made to train ambassadors in the use of the new Metadata Manager tool, as this should simplify the process of registering content and updating metadata records, something of particular importance for new and smaller members. A series of interactive webinars is planned over the course of 2019 to introduce members to the tool and help them get to grips with the functionality. It is envisioned that once ambassadors have familiarity with it, they will also help run these webinars in other languages and time zones.

In May 2018 Crossref hired an Education Manager who is reviewing current documentation, creating a more streamlined and simplified English language support guide. Ambassadors are already involved in the Education Task Force driving this initiative forwards and will provide on-going consultation and feedback.

Continuing expansion of LIVE local events is also planned in 2019. These will focus on areas where Crossref has seen a significant increase of new members as well as identifying areas where uptake is fairly low. Finding partners to work with in these regions to help identify how to support and encourage organizations who are interested in participating will be important, as will the assistance of the ambassador team in maintaining and furthering existing relationships in their regions.

As a community-led organisation, Crossref will continue to 
collaborate with others working in the scholarly communications space. This will help to ensure that both Crossref and the ambassador team stay engaged in wider discussions that impact this dynamic industry.

\section{Conclusion}

The Crossref Ambassador Program is still in its infancy but has had notable success in the first 12 months with concrete results produced. Improvements to enhance communication within the team and greater multi-lingual support will support our efforts to ensure the program continues to develop and support the wider work of Crossref, and of the Ambassadors, going forwards.

\section{Conflict of Interest}

No potential conflict of interest relevant to this article was reported.

\section{Acknowledgments}

Thanks goes to all Crossref Ambassadors for their feedback, in particular to Jae Hwa Chang for her assistance in the production of this article and for being Crossref Ambassador for South Korea.

\section{References}

1. Crossref. Our mission [Internet]. Oxford: Crossref; 2019 [cited 2019 Jan 28]. Available from: https://www.crossref. org/

2. Crossref. Our dashboard [Internet]. Oxford: Crossref; 2019 [cited 2019 Jan 30]. Available from: https://www. crossref.org/dashboard/

3. Crossref. Our people [Internet]. Oxford: Crossref; 2019 [cited 2019 Jan 30]. Available from: https://www.crossref. org/people/

4. Crossref. Our ambassadors [Internet]. Oxford: Crossref; 2019 [cited 2019 Jan 29]. Available from: https://www. crossref.org/community/our-ambassadors/

5. Cormack Wood C. No longer lost in translaton [Internet]. Oxford: Crossref; 2019 [cited 2019 Jan 28]. Available from: https://www.crossref.org/blog/no-longer-lost-in-translation/

6. Crossref. Webinars [Internet]. Oxford: Crossref; 2019 [cited 2019 Jan 28]. Available from: https://www.crossref.org/ webinars/

7. Crossref. Crossref LIVE locals [Internet]. Oxford: Crossref; 2019 [cited 2019 Jan 28]. Available from: https://www. crossref.org/crossref-live-locals/ 